\title{
Distribution functions in the interior of polymer chains
}

\author{
S Redner \\ Center for Polymer Studies and Department of Physics, Boston University, 111 Cum- \\ mington Street, Boston, MA 02215, USA
}

Received 31 January 1980, in final form 14 April 1980

\begin{abstract}
For a polymer chain in a good solvent, we calculate the probability distribution functions between an endpoint and an interior point, and between two interior points, by using exact enumeration to study a lattice self-avoiding walk model. We find that these distribution functions are different from the usual distribution function between endpoints. At small distance scales, the probability of nearest-neighbour contacts between two interior points is smaller than the probability of contact between two endpoints. The contact probability is found to vary with $N$, the number of monomers between the contacts, as $N^{-\left(d+\theta_{2}\right) \nu}$ with $\left(d+\theta_{2}\right) \nu=+2 \cdot 16 \pm 0.20$ on the FCC, and $\left(d+\theta_{2}\right) \nu=+2.95 \pm 0.20$ on the triangular lattice. From this we deduce that the exponent $\theta_{2}$ describing the short-distance spatial decay of the corresponding distribution function is $\theta_{2}=0.67 \pm 0.34$ and $\theta_{2}=$ $1.93 \pm 0.27$ on the FCC and triangular lattices respectively. For large distance scales, we present evidence that the distribution functions vary as $\exp \left(-\left(r / N^{\nu}\right)^{\delta_{i}}\right)$, where $\nu$ is the correlation length exponent, and where the exponent $\delta_{i}$ describes the large-distance spatial decay. On the square lattice we estimate that $\delta_{1}=4.5 \pm 0.4$ for the endpoint-interior distribution, and $\delta_{2}=4.6 \pm 0.6$ for the interior-interior distribution (while $\delta_{0}=4.0$ for the endpoint problem). On the simple cubic lattice, we estimate $\delta_{1}=2.6 \pm 0.06$ (while $\delta_{0}=$ 2.5).
\end{abstract}

\section{Introduction}

Much of our current understanding of the configuration of linear polymer chains in a good solvent is based on the study of the probability distribution function $P_{N}(r)$ (see e.g. de Gennes 1979). This is defined as the probability that one end of a polymer chain consisting of $N$ monomers is located at $r$, given that the other end is at the origin. This function behaves as

$$
P_{N}(r)=N^{-d \nu} f\left(r / N^{\nu}\right)
$$

where $d$ is the spatial dimension, $\nu$ is the correlation length exponent, and the scaling function $f$ varies as $f(z) \sim z^{\theta}$ for $z \ll 1$, and $f(z) \sim \exp \left(-z^{\delta}\right)$ for $z \gg 1$ (see figure 1).

The nature of this distribution function has been extensively investigated. Generally, the self-avoiding walk (SAW) model on a lattice has been employed in order to simulate polymer chains in a good solvent. Fisher (1958) first suggested that unlike the random walk model, the distribution function for SAw's was non-Gaussian. This was subsequently confirmed qualitatively by a Monte Carlo investigation (Wall and Erpenbeck 1959), and by an exact enumeration study (Fisher and Hiley 1961). By deriving more extensive data, Domb et al (1965) were able to study quantitatively the large-distance tail of the distribution function. They found that the exponent $\delta=4.0$ on the square lattice, and that $\delta=2.5$ on the simple cubic lattice. Fisher (1966) used 
(a)
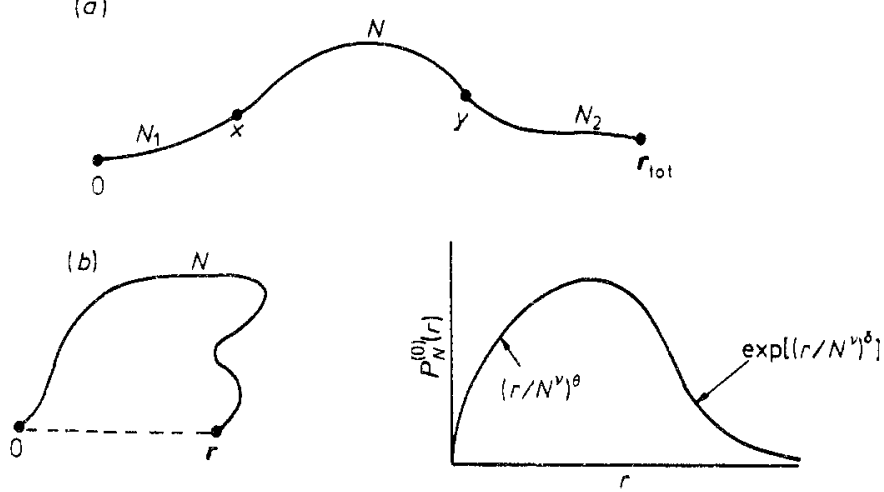

(c)
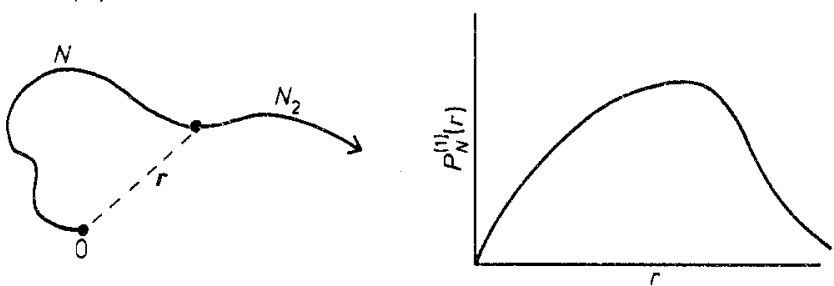

(d)
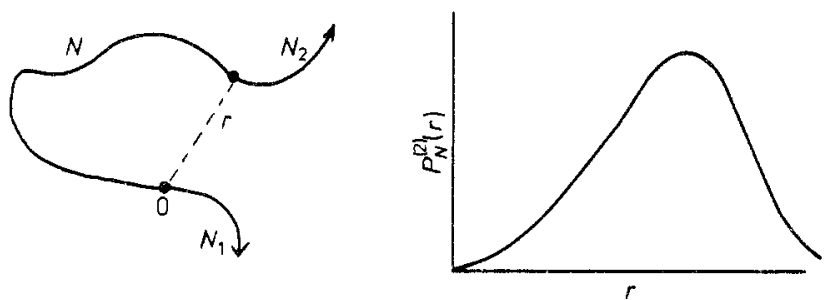

Figure 1. (a) The general chain configuration from which the probability distribution functions considered in this paper are derived. The usual endpoint problem is schematically defined in $(b)$. The distribution function is skerched on the right to illustrate its physical features: it goes to 0 at the origin with infinite slope $(\theta<1)$, and it decays with a power of an exponential for large $r .(c)$ The interior-endpoint problem. The arrow at the end of the chain indicates the limit $N_{2} \rightarrow \infty$. Here, the decay of the distribution function is less steep near the origin than in $(a)$, and the more sharply defined peak is located further to the right. (d) The interior-interior problem. When 0 and $r$ are separated by a single lattice spacing, we obtain the interior contact probability. Here, the distribution function goes to zero near the origin with zero slope (for two dimensions only), and the central peak is much more pronounced.

analytic techniques to express $\delta$ in terms of $\nu$, and found that $\delta=(1-\nu)^{-1}$. McKenzie and Moore (1971) employed a scaling approach to calculate both $\delta$ and $\theta$ in terms of other critical exponents for the polymer problem. McKenzie (1973) later used series techniques to study the detailed behaviour of equation (1).

The exponent $\theta$ gives a measure of the strength of the excluded volume interaction at small distances and is related to the initial ring closure probability, a quantity which has been studied in detail (Martin et al 1966, Sykes et al 1972 a,b and references 
therein). From these studies it was found that $\theta=\frac{4}{9}$ for $d=2$, and that $\theta=\frac{7}{36}$ for $d=3$. More recently, des Cloizeaux (1974) also calculated $\delta$ and $\theta$ based on a Lagrangian field theory for the $n$-vector spin model in the limit $n \rightarrow 0$, a limit which corresponds to the polymer problem (de Gennes 1972). From this, $\theta$ was found to be $(\gamma-1) / \nu$, where $\gamma$ is the polymer analogue of the susceptibility exponent.

The spatial correlations between endpoints have been the primary focus of previous investigations. This reflects, perhaps, the fact that one naturally focuses on the two endpoints. Additionally, in the $n \rightarrow 0$ limit of the usual $n$-vector model, only the endpoints of the chain can be studied. Thus the nature of the spatial correlations between arbitrary points of the chain is still poorly understood. However such correlations are relevant in many experimental studies; for example, in determining the radius of gyration and the hydrodynamic radius of a polymer chain. Therefore it is important to investigate theoretically the nature of the distribution function between arbitrary points of a polymer chain. Recently Schäfer and Witten (1977) have used $\epsilon$-expansion techniques to derive scaling laws for the general correlation functions. Also, Croxton (1979) has employed a new diagrammatic method to study the mean separation of two interior points within a polymer chain. Very recently, des Cloizeaux (1980) has also used the $\epsilon$-expansion to calculate the exponents which govern the short-range behaviour of the distribution functions in three dimensions.

In this article, we use exact enumeration techniques to study quantitatively the probability distribution function between arbitrary points of a polymer chain in both two and three dimensions, and to elucidate the asymptotic behaviour at both small and large distances. Qualitatively, we find that the correlations between two interior points are more 'rigid-rod'-like than that between two endpoints. That is, two interior points are more likely to be separated by their mean distance than are endpoints. This effect reflects additional excluded volume constraints imposed on a monomer within the interior of the chain. The additional interaction manifests itself in the exponents which describe the asymptotic behaviour of the probability distribution functions. The focus of our study is to present evidence that these exponents are different from the exponents of the usual endpoint distribution function.

In the next section, we shall first present a formulation of the problem, due primarily to the work of des Cloizeaux (1980). In $\S 3$, we examine the behaviour of the previously unstudied interior-interior distribution function for small distances. Then in $\$ 4$, we turn to the behaviour of the various distribution functions at large distances. Finally, we present a summary in $\S 5$.

\section{Distribution functions for SAW's}

In studying the correlations between arbitrary points of a polymer chain, des Cloizeaux (1980) has found that these correlations can be simply divided into three classes: endpoint-endpoint, endpoint-interior point, and interior point-interior point. Accordingly, des Cloizeaux introduced new probability distribution functions which correspond to the three classes as follows. Consider a SAW which begins at the origin and goes to $x$ in $N_{1}$ steps, to $y$ in $N$ steps, and finally to $r_{\text {tot }}$ in $N_{2}$ steps. We shall use the probability $P\left(0, x, y, r_{\text {tot }} ; N_{1}, N, N_{2}\right)$ to describe this configuration of $n=N_{1}+N+N_{2}$ steps as indicated in figure $1(a)$. The usual endpoint distribution function can then be defined as (see figure $1(b)$ )

$$
P_{N}^{(0)}(r)=P(0,0, r, r ; 0, N, 0),
$$


where the superscript 0 refers to the endpoint problem. Similarly, the distribution function between an endpoint and an interior point can be defined as (see figure $1(c)$ )

$$
P_{N}^{(1)}(r)=\lim _{N_{2} \rightarrow \infty} P\left(0,0, r, r_{\text {tot }} ; 0, N, N_{2}\right),
$$

where the superscript 1 refers to the endpoint-interior point problem.

At small distance scales, $(2 a)$ describes the ring closure probability, while $(2 b)$ describes the limited ring closure or 'tadpole' probability. The latter is the probability that a SAw terminates by intersecting with another point of the walk which is not an endpoint. Wall et al (1954) were the first to consider this problem, and they estimated that the ring and tadpole probabilities were approximately equal. Subsequent, more accurate studies by Trueman and Whittington (1972), Guttmann and Sykes (1973) and Whittington et al (1975) found a slight difference between these two probabilities, indicating the possibility that the distribution functions $(2 a)$ and $(2 b)$ are in different universality classes.

Finally, we may define the distribution function between two interior points as (see figure $1(d)$ )

$$
P_{N}^{(2)}(r)=\lim _{N_{1} \rightarrow \infty} \lim _{N_{2} \rightarrow \infty} P\left(0, x, y, r_{\text {tot }} ; N_{1}, N, N_{2}\right),
$$

where $\boldsymbol{r}=\boldsymbol{y}-\boldsymbol{x}$, and the superscript 2 refers to the interior-interior problem.

Des Cloizeaux used the $\epsilon$-expansion to study the small-distance behaviour of these distribution functions, and found that they all have the same scaling form, $P_{N}^{(i)}(\boldsymbol{r}) \sim$ $N^{-d \nu} f^{(i)}\left(r / N^{\nu}\right)$, with $f^{(i)}(z) \sim z^{\theta_{i}}$ for $z \ll 1$, with the exponents $\theta_{i}$ being different. For $d=3$, his calculation gives $\theta_{0}=0.273 \pm 0.004, \theta_{1}=0.459 \pm 0.003$ and $\theta_{2}=0.71 \pm 0.05$ (see table 1). That is, the relative importance of the excluded volume interaction near the origin depends on the type of problem being considered. The series for determining $\theta_{0}$ and $\theta_{1}$ have already been derived (Martin et al 1966, Sykes et al $1972 \mathrm{a}, \mathrm{b}$, Guttmann and Sykes 1973), and in the next section we calculate the series for the short-distance limit of the interior-interior distribution function in order to determine $\theta_{2}$.

\section{Small-distance behaviour}

To probe the distribution function between interior points at small distances $\left(r \ll N^{\nu}\right)$, we require the analogue of the ring (or tadpole) closure probability. More correctly, we do not want the closure probability, but rather the probability for forming a nearestneighbour contact. Because this quantity depends only on the scaling variable $r / N^{\nu}$, finding the $N$-dependence of the probability for fixed spatial separation of the contact also gives its $r$-dependence. We therefore consider $P\left(0, x, y, r_{\text {tot }}, N_{1}, N, N_{2}\right)$ for $|\boldsymbol{y}-\boldsymbol{x}|=l$, where $l$ is a single lattice spacing. This gives the probability that two points separated by $N$ bonds within an $n$-step sAw are nearest neighbours. We call this quantity the 'interior contact' probability $p_{n}(N)$, and this is the analogue of the contact probabilities in the ring and tadpole problems. In fact, all three contact probabilities are appropriate cases of the interior-interior distribution function at short distances. For example, if $N_{1} / N \rightarrow 0$ or $N_{2} / N \rightarrow 0$, a crossover to the probability of tadpole formation occurs. Moreover in the limit that both $N_{1}$ and $N_{2} \rightarrow 0$, we recover simply the ring closure probability.

For our calculation, we choose $N_{1}=N_{2}$ whenever possible. This choice minimises the crossover effects due to the tadpole problem. Sometimes we are obliged to choose 
Table 1. Summary of current estimates for the short- and large-distance decay exponents for the three probability distribution functions considered in this work.

\begin{tabular}{|c|c|c|}
\hline & $d=2$ & $d=3$ \\
\hline$\theta_{0}$ & $\frac{4 a, b}{9}$ & $\begin{array}{l}\frac{7}{36}{ }^{\mathrm{a}} \\
0.275 \pm 0.002^{\mathrm{b}} \\
0.273 \pm 0.004^{\mathrm{c}^{\prime}}\end{array}$ \\
\hline$\theta_{1}$ & $\begin{array}{l}0.84 \pm 0 \cdot 01^{d} \\
0.84 \pm 0 \cdot 13^{e}\end{array}$ & $\begin{array}{l}0.61 \pm 0.17^{\mathrm{e}} \\
0.70 \pm 0.12^{\mathrm{f}} \\
0.459 \pm 0.003^{\mathrm{c}}\end{array}$ \\
\hline$\theta_{2}$ & $\begin{array}{l}2 \cdot 0^{c^{\prime}} \\
1.93 \pm 0 \cdot 27^{i}\end{array}$ & $\begin{array}{l}0.71 \pm 0.05^{\circ} \\
0.67 \pm 0.34^{i}\end{array}$ \\
\hline$\delta_{0}$ & $4 \cdot 0^{\mathrm{g}, \mathrm{h}}$ & $2 \cdot 5^{\mathrm{g}, \mathrm{h}}$ \\
\hline$\delta_{1}$ & $4 \cdot 5 \pm 0 \cdot 4^{i}$ & $2 \cdot 6 \pm 0.06^{\mathrm{i}}$ \\
\hline$\delta_{2}$ & $4 \cdot 6 \pm 0 \cdot 6^{i}$ & - \\
\hline \multicolumn{3}{|c|}{ 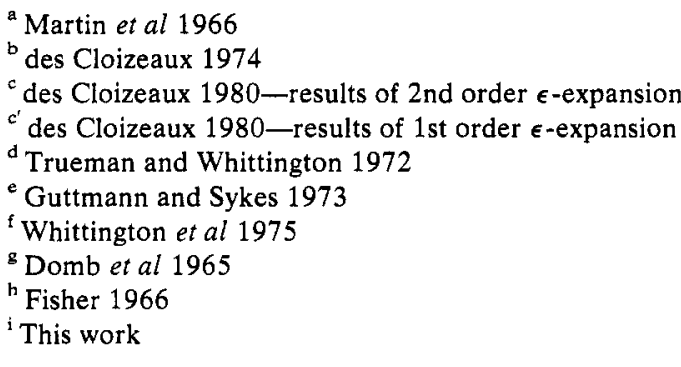 } \\
\hline
\end{tabular}

$N_{1}=N_{2}+1$, when $n=N_{1}+N+N_{2}$ is an odd number and $N$ is even (or vice versa). This condition introduces some even-odd oscillations in our series, but this is not a serious problem. Additionally, we consider close-packed lattices only, where $N$ can be both even or odd. On loose-packed lattices, $N$ can be odd only (when $l$ is a single lattice spacing), and even though we may enumerate longer walks, the corresponding data are not sufficiently well-behaved to give predictions as accurate as those on close-packed lattices.

Our data for the triangular and FCC lattices are given in table 2. Each row corresponds to a fixed number of total bonds, $n$, and each column corresponds to a fixed number of bonds, $N$, between the contact. The entries thus give the number of SAw's with an interior contact in the middle of the walk for fixed $n$ and $N$. Other nearestneighbour contacts may also occur, but they are not of relevance for the problem considered here. From the tables, we obtain the interior contact probability $p_{n}(N)$ by dividing each entry by the total number of SAw's of that order. We then extrapolate this probability to $n \rightarrow \infty$, while $N$ remains fixed, in order to obtain the asymptotic contact probability $p(N)$ between two interior points within an infinitely long chain (last row of each table). We accomplish this by using Neville tables to perform linear, quadratic, cubic, etc extrapolations based on alternate pairs of data points. This type of extrapolation is called for because the series derived from considering both interior segments in the centre of a chain, and those offset by one bond from the centre, exhibit even-odd oscillations. For the first few $N$, there are sufficient terms in the series for $p_{n}(N)$ to give quite accurate asymptotic estimates. However the series are progres- 
Table $2(a)$. Self-avoiding walk data on the triangular lattice. Each row corresponds to a given number of total bonds in the walk (beginning at $n=1$ ). The $N$ th column gives the number of walks in which there are $N$ bonds between an interior contact occurring in the 'middle' of the walk. Here 'middle' means that the contact occurs exactly in the centre of the walk if $n$ and $N$ are both even or both odd, while the contact is offset by one bond from the centre otherwise. The first column is the total number of all walks, and the upper right diagonal is the total number of ring closures of $n+1$ steps. The lower portion of the table is the continuation of the table to the right. "The closure probability $p_{n}(N)$ is found by dividing each entry by the total number of walks of that order. The bottom row then gives the extrapolated value of this closure probability, $p(N)=\lim _{n \rightarrow \infty} p_{n}(N)$.

\begin{tabular}{|c|c|c|c|c|c|c|c|}
\hline$n / N$ & 1 & 2 & 3 & 4 & 5 & 6 & 7 \\
\hline 1 & 6 & & & & & & \\
\hline 2 & 30 & 12 & & & & & \\
\hline 3 & 138 & 48 & 24 & & & & \\
\hline 4 & 618 & 180 & 84 & 60 & & & \\
\hline 5 & 2730 & 792 & 264 & 192 & 180 & & \\
\hline 6 & 11946 & 3444 & 1128 & 528 & 552 & 588 & \\
\hline 7 & 51882 & 15000 & 4728 & 2196 & 1416 & 1728 & 1968 \\
\hline 8 & 224130 & 64932 & 20304 & 8928 & 5616 & 4236 & 5676 \\
\hline 9 & 964134 & 280200 & 86496 & 37776 & 21576 & 16152 & 13692 \\
\hline 10 & 4133166 & 1204572 & 369732 & 158160 & 90480 & 59004 & 49968 \\
\hline 11 & 17668938 & 5159448 & 1573608 & 670632 & 374928 & 245688 & 172908 \\
\hline 12 & 75355206 & 22043292 & 6703068 & 2828724 & 1580256 & 1009500 & 712380 \\
\hline 13 & 320734686 & 93952428 & 28474704 & 11977356 & 6620904 & 4233528 & 2891640 \\
\hline \multirow[t]{2}{*}{14} & 1362791250 & 399711348 & 120922272 & 50553456 & 27902556 & 17637600 & 12073308 \\
\hline & $p(N)$ & $\begin{array}{r}=0.2982 \\
\pm 6\end{array}$ & $\begin{array}{r}0.0882 \\
\pm 6\end{array}$ & $\begin{array}{r}0.0345 \\
\pm 7\end{array}$ & $\begin{array}{r}0.0184 \\
\pm 8\end{array}$ & $\begin{array}{r}0.0115 \\
\pm 10\end{array}$ & $\begin{array}{r}0.0077 \\
\pm 10\end{array}$ \\
\hline$n / N$ & 8 & 9 & 10 & 11 & 12 & 13 & 14 \\
\hline 8 & 6840 & & & & & & \\
\hline 9 & 19512 & 24240 & & & & & \\
\hline 10 & 46932 & 68700 & 87252 & & & & \\
\hline 11 & 164988 & 165840 & 246840 & 318360 & & & \\
\hline 12 & 543948 & 565668 & 599952 & 900432 & 1173744 & & \\
\hline 13 & 2212308 & 1795920 & 1998456 & 2204508 & 3323376 & 4366740 & \\
\hline \multirow[t]{3}{*}{14} & 8849472 & 7187256 & 6168444 & 7215720 & 8194560 & 12385836 & 4366740 \\
\hline & 0.0050 & 0.0035 & & & & & \\
\hline & \pm 10 & \pm 18 & & & & & \\
\hline
\end{tabular}

sively shorter for larger $N$ and the uncertainties associated with extrapolation correspondingly increase. The error bars given in the last row of the tables represent subjective estimates of the uncertainties based on the Neville table analysis.

Next we need to find the dependence of the probability, $p(N)$, on $N$. According to equation (1), this probability should vary as $N^{-\left(d+\theta_{2}\right) v}$. Therefore we plot $p(N)$ versus $N$ on a double logarithmic scale (see figure 2) and use a least-squares fit to determine the slope of the straight line which best fits the data points. We estimate the error associated with this slope by the following procedure. First we successively delete the first few data points, and calculate the slope (by least squares) of the straight line which best fits these subsets of data points. The variation of the slopes between subsets 
Table $2(b)$. The same SAW data as in $(a)$ for the FCC lattice.

\begin{tabular}{|c|c|c|c|c|c|}
\hline$n / N$ & 1 & 2 & 3 & 4 & 5 \\
\hline 1 & 12 & & & & \\
\hline 2 & 132 & 48 & & & \\
\hline 3 & 1404 & 480 & 264 & & \\
\hline 4 & 14700 & 4656 & 2496 & 1680 & \\
\hline 5 & 152532 & 47760 & 22800 & 15624 & 11640 \\
\hline 6 & 1573716 & 485904 & 229368 & 133440 & 102936 \\
\hline 7 & 16172148 & 4972032 & 2287896 & 1321008 & 874272 \\
\hline 8 & 165697044 & 50692272 & 23218344 & 12955296 & 8541048 \\
\hline 9 & 1693773924 & 517215774 & 234741432 & 130570128 & 82600248 \\
\hline \multirow[t]{2}{*}{10} & 17281929564 & 5265877872 & 2384687064 & 1310778864 & 827100192 \\
\hline & $p(N)$ & $\begin{array}{r}=0.3010 \\
\pm 10\end{array}$ & $\begin{array}{r}0.1325 \\
\pm 10\end{array}$ & $\begin{array}{r}0.0698 \\
\pm 20\end{array}$ & $\begin{array}{r}0.0430 \\
\pm 20\end{array}$ \\
\hline$n / N$ & 6 & 7 & 8 & 9 & 10 \\
\hline 6 & 86352 & & & & \\
\hline 7 & 751200 & 673104 & & & \\
\hline 8 & 6269136 & 5789472 & 5424768 & & \\
\hline 9 & 60512064 & 47765256 & 46292256 & 44828400 & \\
\hline \multirow[t]{3}{*}{19} & 577866000 & 426253080 & 379082784 & 380448408 & 377814528 \\
\hline & 0.0280 & 0.0210 & & & \\
\hline & \pm 30 & \pm 40 & & & \\
\hline
\end{tabular}

indicates the presence and magnitude of possible systematic error. In addition, we also find the slopes for data in which selected points have been moved to the edge of an error bar. Combining these two sources of uncertainties gives confidence limits of the size of the error. Thus we estimate that $\left(d+\theta_{2}\right) \nu=+2 \cdot 16 \pm 0 \cdot 20$ on the FCC lattice, and $\left(d+\theta_{2}\right) \nu=+2.95 \pm 0.20$ on the triangular lattice. Using the estimate of 0.588 for $\nu$ in three dimensions (Le Guillou and Zinn-Justin 1977) leads to $\theta_{2}=0.67 \pm 0.34$ on the FCC lattice. On the triangular lattice, we use the Flory value of 0.75 for $\nu$ to yield $\theta_{2}=1.93 \pm 0 \cdot 27$.

The three-dimensional result is in good agreement with the prediction of $\theta_{2}=0.71$ from the second-order $\epsilon$-expansion of des Cloizeaux (1980). In two dimensions the second-order expansion is poorly behaved, although the first-order term gives $\theta_{2}=2$ in close agreement with our estimate. It is also interesting that the $N$ dependence of the interior contact probability is very different from the $N$ dependence of the ring and tadpole probabilities in $d=2$ (although for $d=3$, the contact probabilities have nearly the same $N$ dependence). This shows that excluded volume effects are very strong when two interior points are nearby, even more so than when an interior point and an endpoint are nearby.

\section{Large-distance behaviour}

To obtain a qualitative understanding of the properties at large distance scales $\left(r \gg N^{\nu}\right)$, let us consider the case $N=7$ for the three distribution functions on the square lattice 


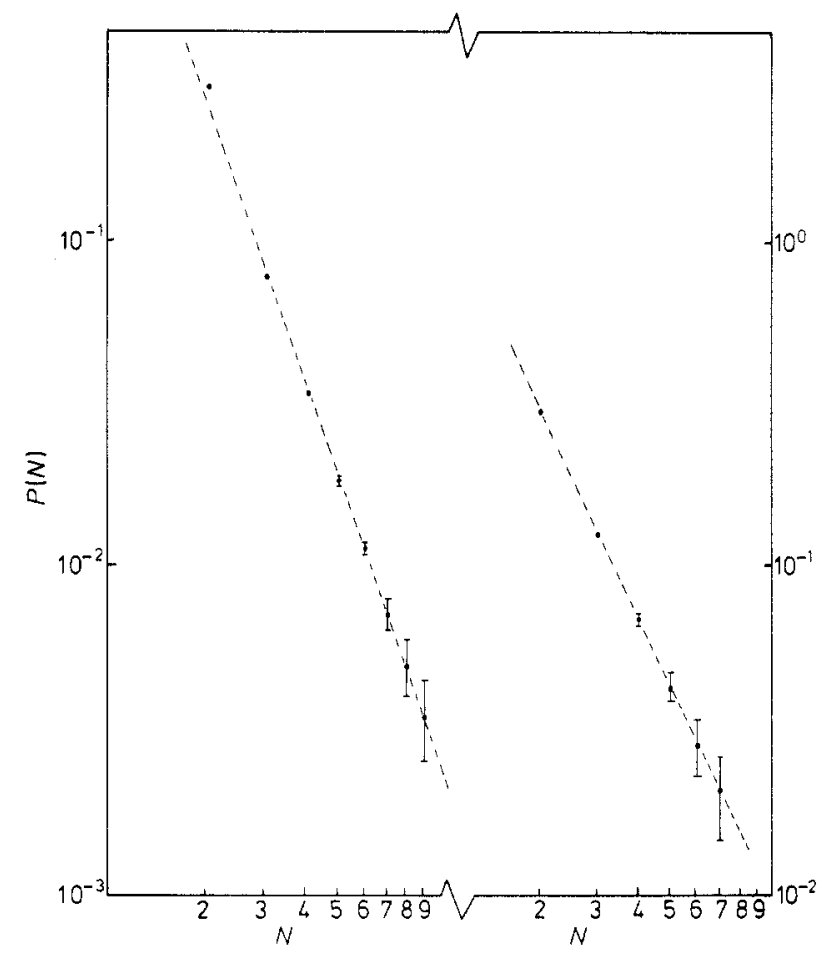

Figure 2. A plot of $\log p(N)$ versus $\log N$ in order to estimate the exponent $\left(d+\theta_{2}\right) \nu$. The left-hand portion shows data from the triangular lattice, and a linear least-squares fit (shown dashed) yields the estimate $\left(d+\theta_{2}\right) \nu=+2.95 \pm 0 \cdot 20$. The right-hand portion shows data from the FCC lattice. Here the least-squares fit yields the estimate $\left(d+\theta_{2}\right) v=+2 \cdot 16 \pm 0 \cdot 20$.

(see figure 3). To define $P_{7}^{(1)}(\boldsymbol{r})$, we consider all 14-step SAw's, and ask for the probability distribution between one end and the midpoint. The midpoint is the most natural choice for defining $P_{N}^{(1)}(\boldsymbol{r})$, although in the $N \rightarrow \infty$ limit, any interior point could equally well be chosen as long as $N_{1} / N$ does not approach zero. However, for the relatively short chains considered here, choosing the interior point close to the end of the chain gives behaviour indicative of the endpoint problem. Therefore to minimise such crossover effects, we have defined $P_{N}^{(1)}(r)$ using an endpoint and the midpoint.

Similarly, to define $P_{7}^{(2)}(\boldsymbol{r})$, we consider all 21 -step saw's, and examine the probability distribution between two points which trisect the chain. Again there is considerable freedom in choosing the two interior points, and our choice minimises the various crossover effects mentioned previously.

In addition to $P_{N}^{(i)}(\boldsymbol{r})$, we have also considered $\tilde{P}_{N}^{(i)}(x)$, the distribution function for the probability that the absolute value of one cartesian coordinate of $r$ equals $x$. One advantage of this distribution function is that it is smoother than $P_{N}^{(i)}(r)$, and is therefore more suited to visual inspection (see figure 4). The use of $\tilde{P}^{(i)}$ presumes that the radial distribution function is spherically symmetric, so that the projection of this function onto one coordinate axis will give unbiased results. The spherical symmetry of $\tilde{P}^{(0)}$ has been previously established by Domb et al (1965), and a priori, there is no reason to doubt that $\tilde{P}^{(1)}$ and $\tilde{P}^{(2)}$ are not also spherically symmetric since there is no preferred spatial direction. Consequently we have assumed this result for the distribution functions in the analysis that follows. 

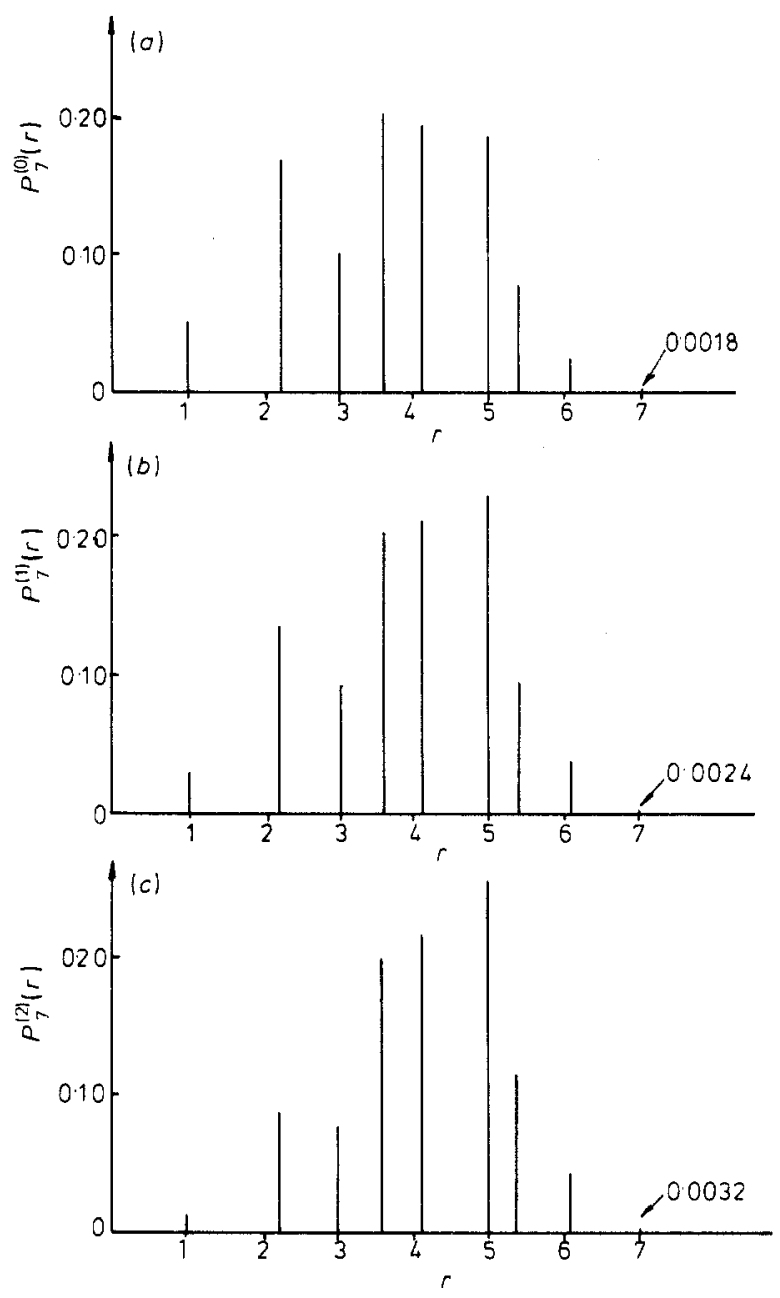

Figure 3. The radial probability distribution functions on the square lattice for the three problems defined in figure 1: $(a)$ endpoint-endpoint, $(b)$ endpoint-interior, $(c)$ interiorinterior. Data are from the square lattice, and there are seven bonds between the origin and $r$. Notice that the data exhibit the qualitative features outlined in figure 1 .

For the endpoint problem, the earlier study of Domb et al was based on fitting $\tilde{P}^{(0)}$ to

$$
A \exp \left[-\left(x / N^{\nu}\right)^{\delta_{0}}\right]
$$

to find the exponent $\delta_{0}$ describing the decay of the distribution function at large distances. On the square lattice, there is a slight dip at the origin in $\tilde{P}^{(0)}$ which is not accounted for by the exponential (see figure 4). This was not judged to be serious for the endpoint problem, but for the other two problems, the dip near the origin is much more prominent. As a result, a simple exponential is not adequate to describe the data for all $r$. We have therefore analysed the radial distribution functions, in order properly to account for the anomalous small-distance behaviour. Thus instead of using $(3 a)$, we have attempted to fit our data to the function

$$
P_{N}^{(i)}(r) \sim A\left(r / N^{\nu}\right)^{\theta_{i}} \exp \left[-\left(r / N^{\nu}\right)^{\delta_{i}}\right]
$$



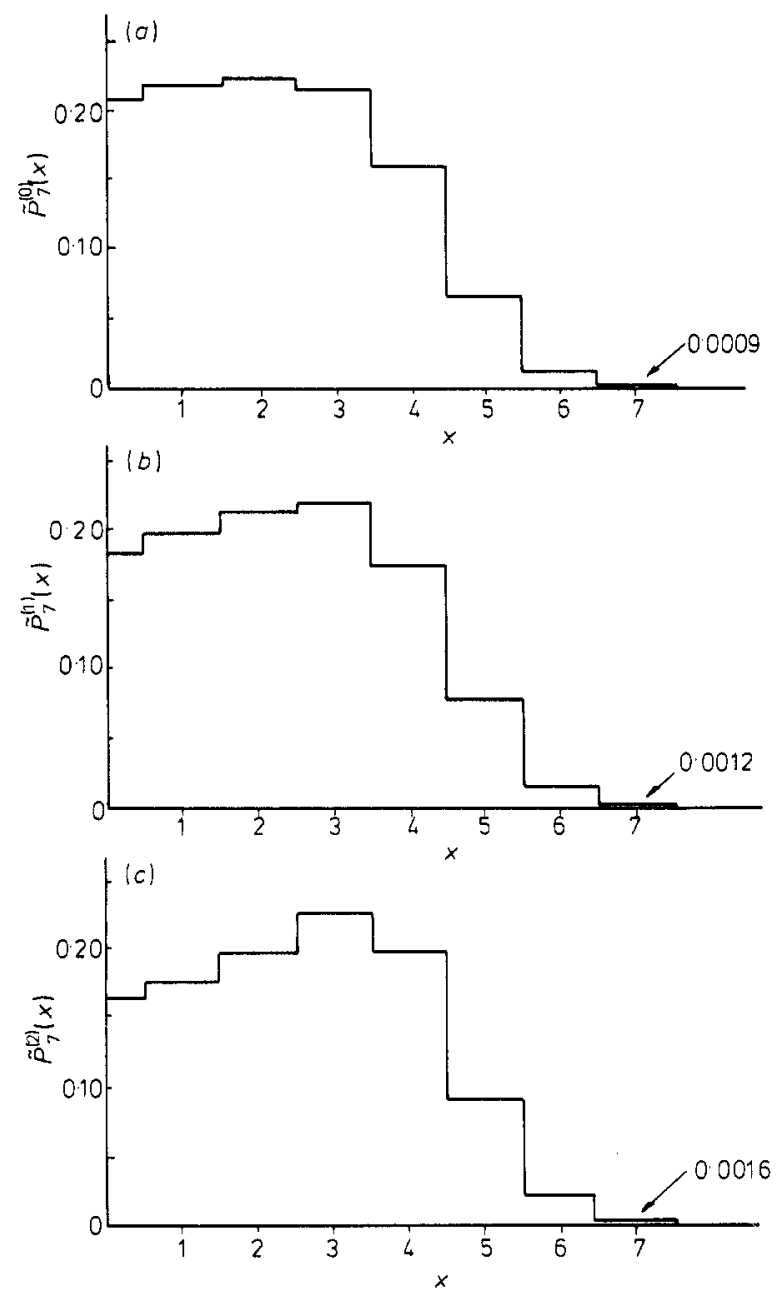

Figure 4. The projection of the data of figure 3 onto one cartesian coordinate. This defines the cartesian probability distribution function $\tilde{P}_{N}^{(i)}(x)$ for the three problems $(a),(b)$ and $(c)$. For these cases, the relative magnitudes of the dips at the origin (ratio of peak height to height at the origin) are $6.7 \%, 12.4 \%$ and $27.5 \%$ respectively.

where $\delta_{i}$ now describes the decay of $P_{N}^{(i)}(\boldsymbol{r})$ at large distances. While each factor is valid only in one regime (either $r \ll N^{\nu}$, or $r \gg N^{\nu}$ ), the product fits the data quite well for all $r$. Furthermore, in the region where one factor is rapidly varying; the other is virtually constant. Thus for $r \ll N^{\nu}$, $\exp \left[-\left(r / N^{\nu}\right)^{\delta_{i}}\right] \approx 1$, while for $r \gg N^{\nu}$, the decay is purely exponential with only a power law prefactor. Consequently, the effects introduced by the influence of the slowly varying factor on the more rapidly varying one should be small.

In order to calculate the exponents $\delta_{1}$ and $\delta_{2}$, we have considered all SAW's of up to 21 bonds on the square lattice, and up to 14 bonds on the simple cubic lattice. Close-packed lattices were not considered here because it was not possible to obtain series of sufficient length to extrapolate with any confidence. From our enumeration on the square lattice, we have calculated $P^{(0)}$ for $N=1,2, \ldots, 20, P^{(1)}$ and $\tilde{P}^{(1)}$ for 
Table 3. Self-avoiding walk data on the square lattice. Walks are classified according to the absolute value of the vector joining an endpoint with the midpoint of the walk. Only the values for $|x| \geqslant|y|$ are shown.

\begin{tabular}{|c|c|c|c|c|c|c|c|c|c|}
\hline No. of bonds & $x$ & $y$ & $n(x, y)$ & $x$ & $y$ & $n(x, y)$ & $x$ & $y$ & $n(x, y)$ \\
\hline 2 & 1 & 0 & 6 & & & & & & \\
\hline \multirow[t]{2}{*}{4} & 1 & 1 & 56 & & & & & & \\
\hline & 2 & 0 & 22 & & & & & & \\
\hline \multirow[t]{3}{*}{6} & 1 & 0 & 64 & & & & & & \\
\hline & 2 & 1 & 276 & & & & & & \\
\hline & 3 & 0 & 50 & & & & & & \\
\hline \multirow[t]{3}{*}{8} & 1 & 1 & 800 & 3 & 1 & 1032 & & & \\
\hline & 2 & 0 & 636 & 4 & 0 & 138 & & & \\
\hline & 2 & 2 & 1504 & & & & & & \\
\hline \multirow[t]{3}{*}{10} & 1 & 0 & 1196 & 3 & 2 & 6896 & & & \\
\hline & 2 & 1 & 6248 & 4 & 1 & 3572 & & & \\
\hline & 3 & 0 & 3760 & 5 & 0 & 378 & & & \\
\hline \multirow[t]{3}{*}{12} & 1 & 1 & 20408 & 3 & 1 & 42372 & 4 & 2 & 28340 \\
\hline & 2 & 0 & 13612 & 3 & 3 & 37280 & 5 & 1 & 11752 \\
\hline & 2 & 2 & 37472 & 4 & 0 & 17780 & 6 & 0 & 1030 \\
\hline \multirow[t]{4}{*}{14} & 1 & 0 & 35636 & 4 & 1 & 239208 & 5 & 2 & 108630 \\
\hline & 2 & 1 & 163796 & 4 & 3 & 177148 & 6 & 1 & 37376 \\
\hline & 3 & 0 & 109060 & 5 & 0 & 74220 & 7 & 0 & 2790 \\
\hline & 3 & 2 & 239628 & & & & & & \\
\hline \multirow[t]{5}{*}{16} & 1 & 1 & 634480 & 4 & 0 & 728772 & 6 & 0 & 285620 \\
\hline & 2 & 0 & 404792 & 4 & 2 & 1433436 & 6 & 2 & 392640 \\
\hline & 2 & 2 & 1128560 & 4 & 4 & 953024 & 7 & 1 & 115872 \\
\hline & 3 & 1 & 1238448 & 5 & 1 & 1177336 & 8 & 0 & 7534 \\
\hline & 3 & 3 & 1423632 & & & & & & \\
\hline \multirow[t]{5}{*}{18} & 1 & 0 & 1163784 & 4 & 3 & 8429892 & 6 & 3 & 3121496 \\
\hline & 2 & 1 & 5264464 & 5 & 0 & 4222060 & 7 & 0 & 1039528 \\
\hline & 3 & 0 & 3343328 & 5 & 2 & 7765980 & 7 & 2 & 1368136 \\
\hline & 3 & 2 & 7816800 & 5 & 4 & 4625852 & 8 & 1 & 352528 \\
\hline & 4 & 1 & 8543988 & 6 & 1 & 5251236 & 9 & 0 & 20294 \\
\hline \multirow[t]{7}{*}{20} & 1 & 1 & 21679264 & 4 & 4 & 49301144 & 7 & 1 & 21768160 \\
\hline & 2 & 0 & 13609956 & 5 & 1 & 53344972 & 7 & 3 & 12045504 \\
\hline & 2 & 2 & 37720192 & 5 & 3 & 47499624 & 8 & 0 & 3628184 \\
\hline & 3 & 1 & 41073680 & 5 & 5 & 24804832 & 8 & 2 & 4619284 \\
\hline & 3 & 3 & 50985272 & 6 & 0 & 21871168 & 9 & 1 & 1056008 \\
\hline & 4 & 0 & 24584804 & 6 & 2 & 34817560 & 10 & 0 & 54546 \\
\hline & 4 & 2 & 52254172 & 6 & 4 & 20775608 & & & \\
\hline
\end{tabular}

$N=1,2, \ldots, 10$, and $P^{(2)}$ and $\tilde{P}^{(2)}$ for $N=1,2, \ldots, 7$ (see tables 3 and 4 ). On the simple cubic lattice, we have calculated $\tilde{P}^{(1)}$ for $N=1,2, \ldots, 7$ (see table 5). We did not consider $\tilde{P}^{(2)}$ because our data extends only to $N=5$, and this was insufficient to probe asymptotic behaviour.

One way to fit our data with the trial distribution functions defined by $(3 b)$ is to calculate the reduced radial moments

$$
m_{2 k}^{(N)}=\left\langle r_{N}^{2 k}\right\rangle /\left(\left\langle r_{N}^{2}\right\rangle\right)^{k}
$$


Table 4. Classification of SAW's on the square lattice according to the absolute value of the vector joining two points which trisect the chain.

\begin{tabular}{|c|c|c|c|c|c|c|c|c|c|}
\hline No. of bonds & $x$ & $y$ & $n(x, y)$ & $x$ & $y$ & $n(x, y)$ & $x$ & $y$ & $n(x, y)$ \\
\hline 3 & 1 & 0 & 18 & & & & & & \\
\hline 6 & $\begin{array}{l}1 \\
2\end{array}$ & $\begin{array}{l}1 \\
0\end{array}$ & $\begin{array}{l}464 \\
158\end{array}$ & & & & & & \\
\hline 9 & $\begin{array}{l}1 \\
2 \\
3\end{array}$ & $\begin{array}{l}0 \\
1 \\
0\end{array}$ & $\begin{array}{r}860 \\
6036 \\
1238\end{array}$ & & & & & & \\
\hline 12 & $\begin{array}{l}1 \\
2 \\
2\end{array}$ & $\begin{array}{l}1 \\
0 \\
2\end{array}$ & $\begin{array}{l}28240 \\
29732 \\
89832\end{array}$ & $\begin{array}{l}3 \\
4\end{array}$ & $\begin{array}{l}1 \\
0\end{array}$ & $\begin{array}{r}64264 \\
9434\end{array}$ & & & \\
\hline 15 & $\begin{array}{l}1 \\
2 \\
3\end{array}$ & $\begin{array}{l}0 \\
1 \\
0\end{array}$ & $\begin{array}{r}76624 \\
740000 \\
546504\end{array}$ & $\begin{array}{l}3 \\
4 \\
5\end{array}$ & $\begin{array}{l}2 \\
1 \\
0\end{array}$ & $\begin{array}{r}1149776 \\
624356 \\
71038\end{array}$ & & & \\
\hline 18 & $\begin{array}{l}1 \\
2 \\
2\end{array}$ & $\begin{array}{l}1 \\
0 \\
2\end{array}$ & $\begin{array}{r}3699168 \\
3488760 \\
12489312\end{array}$ & $\begin{array}{l}3 \\
3 \\
4\end{array}$ & $\begin{array}{l}1 \\
3 \\
0\end{array}$ & $\begin{array}{r}15555800 \\
16862560 \\
7523056\end{array}$ & $\begin{array}{l}4 \\
5 \\
6\end{array}$ & $\begin{array}{l}2 \\
1 \\
0\end{array}$ & $\begin{array}{r}13045588 \\
5662832 \\
527810\end{array}$ \\
\hline 21 & $\begin{array}{l}1 \\
2 \\
3 \\
3\end{array}$ & $\begin{array}{l}0 \\
1 \\
0 \\
2\end{array}$ & $\begin{array}{r}12272468 \\
107273676 \\
93755008 \\
234656584\end{array}$ & $\begin{array}{l}4 \\
4 \\
5\end{array}$ & $\begin{array}{l}1 \\
3 \\
0\end{array}$ & $\begin{array}{r}257972860 \\
219053104 \\
88916956\end{array}$ & $\begin{array}{l}5 \\
6 \\
7\end{array}$ & $\begin{array}{l}2 \\
1 \\
0\end{array}$ & $\begin{array}{r}137267196 \\
49355976 \\
3879186\end{array}$ \\
\hline
\end{tabular}

for the three distribution functions and extrapolate to $N \rightarrow \infty$ as indicated by the last row in tables 6 and 7. Here $\left\langle r_{N}^{2 k}\right\rangle$ is the mean value of $r^{2 k}$ between the two points under study, and $N$ indicates that the origin and $r$ are separated by $N$ bonds. The series for $m_{2 k}^{(N)}$ again exhibit even-odd oscillations and we therefore use Neville tables based on extrapolating alternate pairs of points to estimate the asymptotic behaviour of $m_{2 k}=$ $\lim _{N \rightarrow \infty} m_{2 k}^{(N)}$. We then compare the results of this extrapolation with the moments derived from $(3 b)$ for various values of $\delta_{i}$. In two dimensions, the reduced moments of the function $(3 b)$ can be expressed in terms of gamma functions as

$$
m_{2 k}=\Gamma\left[\left(2 k+\theta_{i}+2\right) / \delta_{i}\right] / \Gamma\left[\left(\theta_{i}+2\right) / \delta_{i}\right] .
$$

We then vary the trial value of $\delta_{i}$ until the best fit between the two sets of moments is found.

To test whether the procedure described above is valid, we first consider the endpoint distribution function on the square lattice. We use the well-established value $\theta_{0}=\frac{4}{9}$, which follows from the ring closure probability varying as $N^{-11 / 6}$ (Martin et al 1966, Sykes et al $1972 \mathrm{a}, \mathrm{b}$ ), and calculate $m_{2 k}$ from equation (5) for a range of values of $\delta_{0}$. Choosing $\delta_{0}=4$ gives

$$
m_{4}=1.46, \quad m_{6}=2 \cdot 65, \quad m_{8}=5.61, \quad m_{10}=13.35, \quad m_{12}=34.9,
$$

and these numbers give the best fit to the extrapolated moments in table 6(a). Thus, to calculate $\delta_{0}$, one can consider the radial distribution function as well as the cartesian distribution function. This is an extremely useful check for the ensuing analysis.

For the endpoint-interior problem on the square lattice, we use $\theta_{1}=0.84$ in equation (5) -a result which follows from the tadpole probability varying as $N^{-2 \cdot 13}$ 
Table 5. Classification of SAW's on the simple cubic lattice according to the absolute value of the vector joining an endpoint with the midpoint of the walk.

\begin{tabular}{|c|c|c|c|c|c|c|c|c|}
\hline No, of bonds & $x$ & $y$ & $z$ & $n(x, y, z)$ & $x$ & $y$ & $z$ & $n(x, y, z)$ \\
\hline 2 & 1 & 0 & 0 & 30 & & & & \\
\hline \multirow[t]{2}{*}{4} & 1 & 1 & 0 & 192 & & & & \\
\hline & 2 & 0 & 0 & 50 & & & & \\
\hline \multirow[t]{4}{*}{6} & 1 & 0 & 0 & 752 & & & & \\
\hline & 1 & 1 & 1 & 5472 & & & & \\
\hline & 2 & 1 & 0 & 1412 & & & & \\
\hline & 3 & 0 & 0 & 242 & & & & \\
\hline \multirow[t]{3}{*}{8} & 1 & 1 & 0 & 31136 & 2 & 2 & 0 & 13536 \\
\hline & 2 & 0 & 0 & 12280 & 3 & 1 & 0 & 9112 \\
\hline & 2 & 1 & 1 & 52976 & 4 & 0 & 0 & 1170 \\
\hline \multirow[t]{5}{*}{10} & 1 & 0 & 0 & 167384 & 3 & 1 & 1 & 426288 \\
\hline & 1 & 1 & 1 & 1010256 & 3 & 2 & 0 & 108096 \\
\hline & 2 & 1 & 0 & 459520 & 4 & 1 & 0 & 54724 \\
\hline & 2 & 2 & 1 & 632992 & 5 & 0 & 0 & 5602 \\
\hline & 3 & 0 & 0 & 122928 & & & & \\
\hline \multirow[t]{7}{*}{12} & 1 & 1 & 0 & 7419160 & 3 & 3 & 0 & 1028928 \\
\hline & 2 & 0 & 0 & 3942488 & 4 & 0 & 0 & 998952 \\
\hline & 2 & 1 & 1 & 15453744 & 4 & 1 & 1 & 3070224 \\
\hline & 2 & 2 & 0 & 6831632 & 4 & 2 & 0 & 775248 \\
\hline & 2 & 2 & 2 & 9009840 & 5 & 1 & 0 & 314328 \\
\hline & 3 & 1 & 0 & 5103436 & 6 & 0 & 0 & 26746 \\
\hline & 3 & 2 & 1 & 6059808 & & & & \\
\hline \multirow[t]{8}{*}{14} & 1 & 0 & 0 & 43866024 & 3 & 3 & 1 & 67268448 \\
\hline & 1 & 1 & 1 & 277243200 & 4 & 1 & 0 & 47222024 \\
\hline & 2 & 1 & 0 & 139655140 & 4 & 1 & 1 & 20563216 \\
\hline & 2 & 2 & 1 & 239133360 & 4 & 3 & 0 & 8570852 \\
\hline & 3 & 0 & 0 & 56119944 & 5 & 0 & 0 & 7223976 \\
\hline & 3 & 1 & 1 & 193477792 & 5 & 2 & 0 & 5178888 \\
\hline & 3 & 2 & 0 & 81551468 & 6 & 1 & 0 & 1749680 \\
\hline & 3 & 2 & 2 & 100187296 & 7 & 0 & 0 & 127338 \\
\hline
\end{tabular}

(Trueman and Whittington 1972). With the value $\delta_{1}=4 \cdot 5$ this yields for the reduced moments

$m_{4}=1 \cdot 36, \quad m_{6}=2 \cdot 24, \quad m_{8}=4 \cdot 20, \quad m_{10}=8 \cdot 71, \quad m_{12}=19 \cdot 7$,

and these numbers give the best fit to the extrapolation in table $6(b)$. Finally, for the interior-interior problem, we use $\theta_{2}=1.93$ found in the previous section for the triangular lattice, and we assume that this exponent is universal for all two-dimensional lattices. The choice $\delta_{2}=4.6$ yields

$m_{4}=1 \cdot 25, \quad m_{6}=1 \cdot 81, \quad m_{8}=2 \cdot 96, \quad m_{10}=5 \cdot 27, \quad m_{12}=10 \cdot 1$.

These moments are best fits to the extrapolation in table $6(c)$. Thus we conclude that $\delta_{0}=4 \cdot 0$ in agreement with the currently accepted result, and that $\delta_{1}=4 \cdot 5$, and $\delta_{2}=4 \cdot 6$.

We determine a lower bound to the estimates for $\delta_{1}$ and $\delta_{2}$ as follows. We take the values for $\theta_{i}$ to be those at the upper limit of the quoted error bars; that is we take 
Table 6. (a) The reduced radial moments $m_{2 k}$ of the distribution function $P_{N}^{(0)}(\boldsymbol{r})$ on the square lattice, defined by equation (4). The last line gives the extrapolation of each column. (b) The reduced radial moments of the distribution function $P_{N}^{(1)}(r)$ on the square lattice. (c) The reduced radial moments of the distribution function $P_{N}^{(2)}(r)$ on the square lattice.

\begin{tabular}{|c|c|c|c|c|c|}
\hline \multicolumn{6}{|l|}{ (a) } \\
\hline$n$ & \multicolumn{5}{|c|}{$m$} \\
\hline 4 & $1 \cdot 252583$ & 1.786574 & $2 \cdot 796211$ & $4 \cdot 745248$ & 8.665928 \\
\hline 5 & $1 \cdot 297136$ & 1.935567 & $3 \cdot 181652$ & 5.663703 & $10 \cdot 839803$ \\
\hline 6 & $1 \cdot 301491$ & 1.965000 & $3 \cdot 281461$ & 5.934912 & $11 \cdot 517606$ \\
\hline 7 & 1.329399 & 2.062932 & 3.553904 & 6.637389 & $13 \cdot 284960$ \\
\hline 8 & $1 \cdot 331255$ & $2 \cdot 079602$ & $3 \cdot 618281$ & 6.834285 & 13.828458 \\
\hline 9 & $1 \cdot 349514$ & $2 \cdot 146053$ & $3 \cdot 811913$ & $7 \cdot 361467$ & $15 \cdot 230960$ \\
\hline 10 & $1 \cdot 351057$ & $2 \cdot 158885$ & 3.862835 & $7 \cdot 525707$ & $15 \cdot 715202$ \\
\hline 11 & $1 \cdot 363710$ & $2 \cdot 206251$ & 4.005540 & 7.929540 & 16.835737 \\
\hline 12 & $1 \cdot 365010$ & $2 \cdot 216506$ & $4 \cdot 046860$ & 8.067245 & $17 \cdot 259662$ \\
\hline 13 & $1 \cdot 374280$ & $2 \cdot 251967$ & $4 \cdot 156392$ & 8.386175 & $18 \cdot 173079$ \\
\hline 14 & $1 \cdot 375 S 4343$ & $2 \cdot 260226$ & $4 \cdot 190186$ & 8.501634 & 18.539867 \\
\hline 15 & $1 \cdot 382474$ & $2 \cdot 287943$ & $4 \cdot 277417$ & $8 \cdot 761166$ & $19 \cdot 301388$ \\
\hline 16 & $1 \cdot 383328$ & $2 \cdot 294636$ & $4 \cdot 305271$ & 8.858349 & $19 \cdot 617843$ \\
\hline 17 & $1 \cdot 388998$ & $2 \cdot 316952$ & $4 \cdot 376546$ & 9.074048 & $20 \cdot 263000$ \\
\hline 18 & $1 \cdot 389694$ & $2 \cdot 322474$ & $4 \cdot 399865$ & $9 \cdot 156802$ & 20.537816 \\
\hline 19 & $1 \cdot 394314$ & $2 \cdot 340844$ & $4 \cdot 459246$ & $9 \cdot 339019$ & 21.091411 \\
\hline \multirow[t]{2}{*}{20} & $1 \cdot 394890$ & $2 \cdot 345471$ & 4.479039 & $9 \cdot 410265$ & $21 \cdot 331832$ \\
\hline & $1.46 \pm 0.01$ & $2.64 \pm 0.05$ & $5 \cdot 60 \pm 0 \cdot 10$ & $13 \cdot 0 \pm 0 \cdot 5$ & $34 \pm 2$ \\
\hline \multicolumn{6}{|l|}{ (b) } \\
\hline 2 & 1.000000 & 1.000000 & 1.000000 & 1.000000 & 1.000000 \\
\hline 4 & $1 \cdot 118827$ & $1 \cdot 366384$ & $1 \cdot 767517$ & $2 \cdot 364442$ & $3 \cdot 221139$ \\
\hline 6 & $1 \cdot 180592$ & $1 \cdot 523992$ & $2 \cdot 140170$ & $3 \cdot 275234$ & $5 \cdot 416776$ \\
\hline 8 & $1 \cdot 225174$ & 1.690656 & $2 \cdot 549973$ & $4 \cdot 163489$ & $7 \cdot 304960$ \\
\hline 10 & $1 \cdot 249121$ & 1.772191 & $2 \cdot 754872$ & 4.627016 & 8.345361 \\
\hline 12 & $1 \cdot 263946$ & 1.828918 & 2.908831 & 4.995488 & 9.191805 \\
\hline 14 & $1 \cdot 276968$ & 1.876532 & 3.040178 & $5 \cdot 320216$ & 9.958755 \\
\hline 16 & $1 \cdot 286270$ & 1.913140 & $3 \cdot 145912$ & $5 \cdot 592960$ & $10 \cdot 627680$ \\
\hline 18 & $1 \cdot 294448$ & 1.944546 & $3 \cdot 237469$ & 5.834232 & $11 \cdot 234993$ \\
\hline \multirow[t]{2}{*}{20} & $1 \cdot 300866$ & 1.970313 & $3 \cdot 314714$ & 6.043395 & 11.776308 \\
\hline & $1 \cdot 36 \pm 0 \cdot 02$ & $2 \cdot 25 \pm 0 \cdot 05$ & $4 \cdot 15 \pm 0 \cdot 20$ & $8 \cdot 5 \pm 0 \cdot 7$ & $19 \pm 2$ \\
\hline \multicolumn{6}{|l|}{ (c) } \\
\hline 3 & 1.000000 & $1 \cdot 000000$ & 1.000000 & 1.000000 & 1.000000 \\
\hline 6 & $1 \cdot 122063$ & $1 \cdot 382672$ & 1.815435 & 2.475410 & 3.446103 \\
\hline 9 & $1 \cdot 152168$ & 1.461421 & 2.022089 & $3 \cdot 014451$ & $4 \cdot 754586$ \\
\hline 12 & $1 \cdot 177332$ & 1.536555 & $2 \cdot 180665$ & 3.343033 & $5 \cdot 495648$ \\
\hline 15 & $1 \cdot 191058$ & 1.582974 & $2 \cdot 287906$ & 3.561531 & 5.943359 \\
\hline 18 & $1 \cdot 201776$ & $1 \cdot 620940$ & $2 \cdot 381761$ & 3.763094 & $6 \cdot 357080$ \\
\hline \multirow[t]{2}{*}{21} & $1 \cdot 209008$ & 1.647671 & $2 \cdot 451321$ & 3.919540 & $6 \cdot 686247$ \\
\hline & $1.28 \pm 0.03$ & $1.85 \pm 0.07$ & $3 \cdot 0 \pm 0 \cdot 2$ & $5 \cdot 25 \pm 0 \cdot 7$ & $10 \pm 2$ \\
\hline
\end{tabular}

$\theta_{1}=0.84+0.015=0.86$ (Trueman and Whittington 1972) and $\theta_{2}=1.95+0 \cdot 27=2.23$ (our result). Since the reduced moments $m_{2 k}$ calculated from equation (5) decrease when either $\theta_{i}$ or $\delta_{i}$ is increased, we must now use a lower $\delta_{i}$ in order to fit with the extrapolated $m_{2 k}$ given in tables $6(b)$ and $6(c)$. The lowest value of $\delta_{i}$ which gives a calculated $m_{2 k}$ just consistent with the upper limit of the error bars for the extrapolated 
Table 7. The reduced cartesian moments of the distribution function $\tilde{P}_{N}^{(1)}(x)$ on the simple cubic lattice.

\begin{tabular}{|c|c|c|c|c|c|}
\hline \multirow[t]{2}{*}{$n$} & \multicolumn{5}{|c|}{$m$} \\
\hline & $2 \cdot 523864$ & $9 \cdot 491798$ & $43 \cdot 361361$ & 210.687907 & $1040 \cdot 822469$ \\
\hline 6 & 2.463659 & $9 \cdot 182760$ & $43 \cdot 714270$ & 243.446481 & 1491.905589 \\
\hline 8 & $2 \cdot 446661$ & 9.033837 & $43 \cdot 205664$ & 247.297689 & $1608 \cdot 882245$ \\
\hline 10 & $2 \cdot 451683$ & 9.025526 & $43 \cdot 183891$ & $249 \cdot 433290$ & 1659.756523 \\
\hline 12 & $2 \cdot 456470$ & 9.039031 & $43 \cdot 241738$ & 250.568703 & 1682.958140 \\
\hline \multirow[t]{2}{*}{14.} & 2.465084 & 9.085434 & $43 \cdot 510215$ & $252 \cdot 710255$ & $1706 \cdot 359332$ \\
\hline & $2.58 \pm 0.02$ & $10 \cdot 14 \pm 0 \cdot 3$ & $53 \pm 0.3$ & $310 \pm 15$ & $2400 \pm 150$ \\
\hline
\end{tabular}

$m_{2 k}$ yields our lower bound for $\delta_{i}$. This takes into account both the uncertainties in estimating $\theta_{i}$ and extrapolating the reduced moment sequences.

Because the value of $\theta_{1}$ is fairly accurately determined, we therefore estimate that $\delta_{1}=4 \cdot 5 \pm 0 \cdot 4$. For $\delta_{2}$, the shorter reduced moment series and the larger error bars for $\theta_{2}$ combine to yield the estimate $\delta_{2}=4 \cdot 6 \pm 0 \cdot 6$. In summary, our analysis indicates that both $\delta_{1}$ and $\delta_{2}$ are greater than $\delta_{0}$. The higher values of $\delta_{1}$ and $\delta_{2}$ over $\delta_{0}$ are consistent with the interpretation that the effect of the remainder of the chain is to provide a net inward force on the segment between 0 and $r$ at large distance scales only.

For $d>2$, it is easier for the remainder of the chain to penetrate into the region occupied by the segment between 0 and $r$, and hence the relative magnitude of the inward force is decreased. Hence the difference between $\delta_{0}, \delta_{1}$ and $\delta_{2}$ should be reduced, and on the simple cubic lattice we present evidence that this appears to be the case. Additionally, on this lattice the problems associated with the analysis of the radial distribution function can be avoided. Here, the cartesian distribution function does not have a dip near the origin, owing to weaker excluded volume effects (see figure 5). Consequently, we have analysed the cartesian function, and now fit the decay of the distribution to the simple exponential $\tilde{P}_{N}^{(1)}(x) \sim A \exp \left[-\left(x / N^{\nu}\right)^{\delta_{1}}\right]$. Thus our analysis for $\delta_{1}$ is no longer influenced by possible errors in $\theta_{1}$. Choosing $\delta_{1}=2.6$ in (6) gives for the reduced moments

$m_{4}=2 \cdot 58, \quad m_{6}=10 \cdot 1, \quad m_{8}=52 \cdot 1, \quad m_{10}=327, \quad m_{12}=2408$,

and these are a good fit for the extrapolated values quoted in table 7 . Here our only source of error stems from extrapolating the series for $m_{2 k}$. Although the series are relatively short, we are reasonably confident that our estimate for $\delta_{1}$ is accurate to within $0 \cdot 06$.

\section{Summary}

We have studied the probability distribution functions between two endpoints, an endpoint and an interior point, and two interior points for self-avoiding walks in both two and three dimensions. For small distance scales we consider the correlations between interior points, and define an appropriate 'interior contact' probability. From the form of the scaling function $f$ defined by equation (1) this probability should vary with $N$ as $N^{-\left(d+\theta_{2}\right) \nu}$. We estimate from our data that $\left(d+\theta_{2}\right) \nu=+2 \cdot 16 \pm 0 \cdot 20$ on the FCC lattice, leading to $\theta_{2}=0.67 \pm 0.34$ for the exponent describing the decay of the 

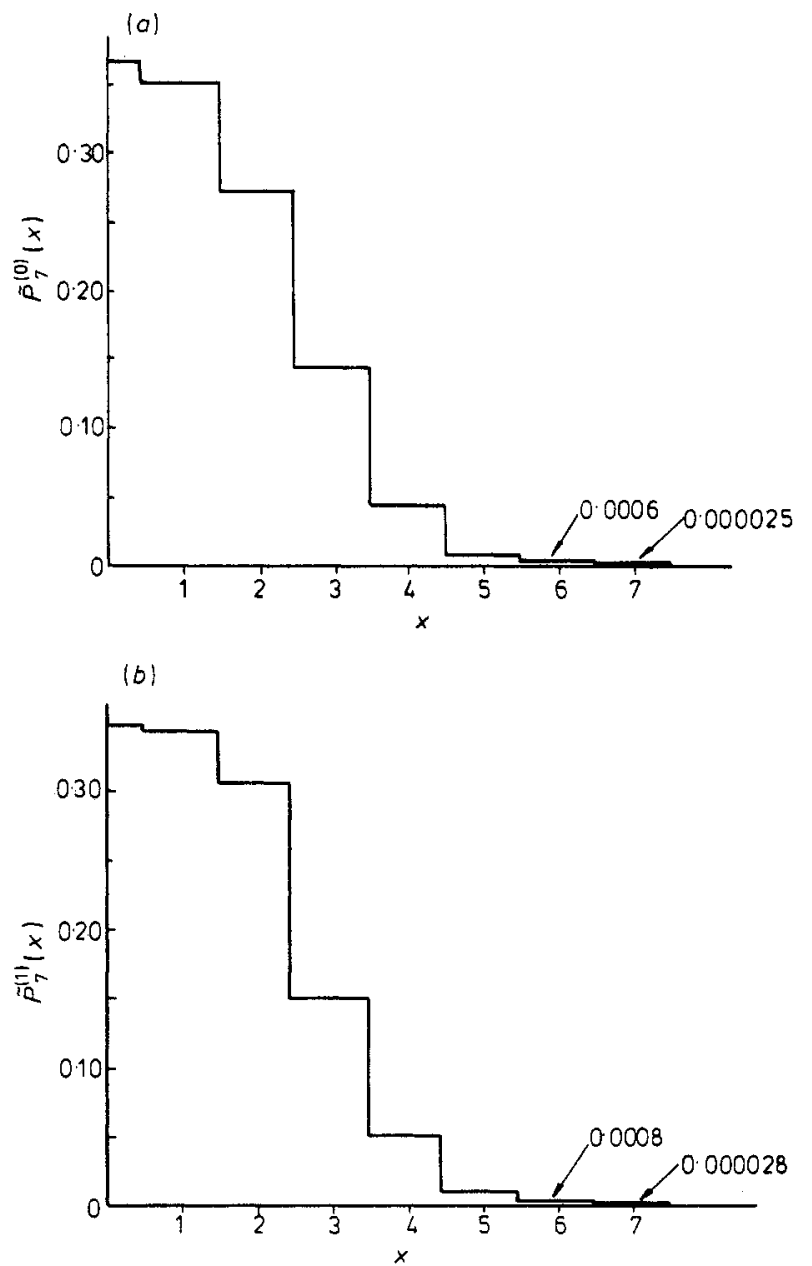

Figure 5. The cartesian probability distribution function for SAw's on the simple cubic lattice. Part (a) shows the endpoint problem for 7-step walks, and part (b) shows the endpoint-interior problem for 14-step walks.

distribution function at small distances. On the triangular lattice, we estimate that $\left(d+\theta_{2}\right) \nu=+2 \cdot 95 \pm 0 \cdot 20$, corresponding to $\theta_{2}=1 \cdot 93 \pm 0 \cdot 27$. These values indicate that the probability of a nearest-neighbour contact between two monomers within the interior of a chain is much less likely to occur than between two endpoints separated by the same number of monomers.

For large $r$, we find that the tails of the distribution functions vary as $\exp \left[-\left(r / N^{\nu}\right)^{\delta_{i}}\right]$. On the square lattice, we estimate that $\delta_{1}=4 \cdot 5 \pm 0 \cdot 4$, and $\delta_{2}=4 \cdot 6 \pm 0 \cdot 6$ (compared with $\delta_{0}=4.0$ for the endpoint distribution function). On the simple cubic lattice, we estimate $\delta_{1}$ to be $2 \cdot 6 \pm 0 \cdot 06$ (compared with $\delta_{0}=2 \cdot 5$ ). The possible higher values for $\delta_{1}$ and $\delta_{2}$ over $\delta_{0}$ indicate that it is less probable for an $N$-step segment in the interior of a longer chain to be in a stretched configuration than it is for a complete chain of $N$ monomers. Coupled with the information at small distances, it appears that the correlations between monomers in the interior of a polymer chain are more rigid-rodlike in character than the correlations between end monomers. 


\section{Acknowledgments}

I am grateful to $\mathrm{M}$ Daoud and $\mathrm{T}$ Witten for many stimulating discussions concerning this work. I wish to thank $H$ Nakanishi and P J Reynolds for a critical reading of the manuscript, and valuable suggestions. I am especially indebted to the Boston University Computing Center for generously providing the necessary computer time, and related facilities for the completion of this project. I also thank the referees for helpful and constructive criticism. This work has been supported in part by the ARO, AFOSR, and by a grant from the Boston University Graduate School of Science.

\section{References}

des Cloizeaux J 1974 Phys. Rev. A 10 1665-9

1980 J. Physique 41 223-28

Croxton C A 1979 J. Phys. A: Math. Gen. 12 2487-95

Domb C, Gillis J and Wilmers G 1965 Proc. Phys. Soc. 85 625-45

Fisher M E 1958 Disc. Faraday Soc. 25200

$1966 \mathrm{~J}$. Chem. Phys. 44 616-22

Fisher M E and Hiley B J 1961 J. Chem. Phys. 34 1253-67

de Gennes P G 1972 Phys. Lett. 38A 339 40

1979 Scaling Concepts in Polymer Physics (Ithaca, NY: Cornell University Press)

Guttmann A J and Sykes M F 1973 J. Phys. C: Solid St. Phys. 6 945-54

Le Guillou J C and Zinn-Justin J 1977 Phys. Rev. Lett. 39 95-8

Martin J L, Sykes M F and Hioe F T 1966 J. Chem. Phys. 46 3478-81

McKenzie D S 1973 J. Phys. A: Math., Nucl. Gen. $8338-52$

McKenzie D S and Moore M A 1971 J. Phys. A: Gen. Phys. 4 L82-6

Schäfer L and Witten T A Jr 1977 J. Chem. Phys. 66 2121-30

Sykes M F, Guttmann A J, Watts M G and Roberts P D 1972a J. Phys. A: Gen. Phys. 5 653-60

Sykes M F, McKenzie D S, Watts M G and Martin J L 1972b J. Phys. A: Gen. Phys. 5 661-6

Trueman R E and Whittington S G 1972 J. Phys. A: Gen. Phys. 5 1664-8

Wall F T and Erpenbeck J J $1959 \mathrm{~J}$. Chem. Phys. 30 634-40

Wall F T, Hiller L A Jr and Wheeler D J 1954 J. Chem. Phys. 22 1036-41

Whittington S G, Trueman R E and Wilker J B 1975 J. Phys. A: Math. Gen. 8 56-60 